\title{
Rituximab maintenance in relapsed or refractory follicular non-Hodgkin's lymphoma: the evidence of its therapeutic value
}

\author{
Kirit M. Ardeshna
}

University College Hospital, London \& Mount Vernon Cancer Centre, Northwood, UK

\begin{abstract}
Introduction: Non-Hodgkin's lymphoma (NHL) is the sixth most common malignancy, and follicular lymphoma (FL) is the second most common form of NHL. FL is generally considered to be incurable, and is characterized by periods of remission followed by episodes of relapse, with median survival of 8-10 years. Maintenance treatment is aimed at improving quality of life and survival.

Aims: To review the current evidence for maintenance rituximab in patients with FL.

Evidence review: Two randomized studies of rituximab maintenance or observation after induction therapy with single-agent rituximab, which were performed mainly in patients with relapsed/refractory disease, have demonstrated a two- to three-fold improvement in median progression-free survival (PFS) in the maintenance arm. Two further studies of rituximab maintenance or observation following induction chemotherapy with or without rituximab performed in patients with relapsed/refractory FL have shown a two- to four-fold increase in median PFS in the maintenance arm. In one of these studies an overall survival benefit has also been demonstrated. An additional study, this time in previously untreated patients, has demonstrated a four-fold improvement in median PFS as well as a significant overall survival benefit with rituximab maintenance following induction with chemotherapy alone.
\end{abstract}

Place in therapy: Currently rituximab maintenance can be considered to be appropriate therapy for patients with relapsed/refractory disease who have not received rituximab previously and who are not suitable for autologous stem cell transplantation, and for patients who receive first-line therapy with chemotherapy without rituximab.

Core Evidence. 2007;2(2):121-129.

Key words: follicular lymphoma, maintenance, refractory, relapsed, rituximab

Core evidence place in therapy summary for rituximab as maintenance therapy in relapsed or refractory non-Hodgkin's lymphoma

\begin{tabular}{|c|c|c|}
\hline Outcome measure & Evidence & Implications \\
\hline \multicolumn{3}{|l|}{ Patient-oriented evidence } \\
\hline $\begin{array}{l}\text { Rituximab maintenance prolongs PFS substantially in patients } \\
\text { with relapsed/refractory FL who are rituximab naïve and respond } \\
\text { to reinduction therapy }\end{array}$ & Clear & Rituximab maintenance improves PFS by approximately two- to three-fold \\
\hline $\begin{array}{l}\text { Rituximab maintenance significantly improves EFS in previously } \\
\text { untreated patients who receive single-agent rituximab as } \\
\text { induction therapy }\end{array}$ & Substantial & EFS increased from 19 to 36 months \\
\hline $\begin{array}{l}\text { Rituximab maintenance improves overall survival in a group of } \\
\text { patients with relapsed/refractory FL who are rituximab naïve } \\
\text { and respond to reinduction with CHOP chemotherapy with or } \\
\text { without rituximab }\end{array}$ & Moderate & $\begin{array}{l}\text { Rituximab maintenance reduces the risk of dying by approximately one-third } \\
\text { at } 3 \text { years }\end{array}$ \\
\hline $\begin{array}{l}\text { In patients with relapsed/refractory } \mathrm{FL} \text {, re-treatment with } \\
\text { rituximab at disease progression may confer a similar period of } \\
\text { rituximab benefit as rituximab maintenance }\end{array}$ & Moderate & $\begin{array}{l}\text { Re-treatment strategy may be less costly but needs verification. RESORT } \\
\text { trial ongoing }\end{array}$ \\
\hline $\begin{array}{l}\text { Rituximab maintenance is beneficial in patients with relapsed/ } \\
\text { refractory FL who have been exposed to prior rituximab }\end{array}$ & None & \\
\hline $\begin{array}{l}\text { Rituximab maintenance is beneficial in previously untreated } \\
\text { patients with FL who receive chemotherapy and rituximab as } \\
\text { induction therapy }\end{array}$ & None & PRIMA study ongoing \\
\hline $\begin{array}{l}\text { Rituximab maintenance results in an improvement in } \\
\text { patients' quality of life }\end{array}$ & None & \\
\hline continued overleaf... & & \\
\hline
\end{tabular}


...table continued

Outcome measure

Evidence

Implications

Economic evidence

Rituximab maintenance in relapsed/refractory patients is cost effective compared with autograft

Rituximab maintenance is cost effective compared with observation alone

Limited

Limited Estimated cost of 1 quality-adjusted life-year is $£ 8910$

CHOP, cyclophosphamide, vincristine, prednisone, doxorubicin; EFS, event-free survival; FL, follicular lymphoma; PFS, progression-free survival.

\section{Scope, aims, and objectives}

The aim of this article is to review the evidence for the use of rituximab (MabThera ${ }^{\circledR}$, F. Hoffmann-La Roche) as maintenance therapy and to determine its place in therapy in the management of patients with relapsed or refractory follicular lymphoma (FL).

\section{Methods}

English language literature searches were conducted on January 24, 2007 in the following databases, searching from the beginning of the database to date unless otherwise stated. The search strategy was "rituximab AND low grade lymphoma" unless otherwise stated:

- PubMed, http://www.ncbi.nlm.nih.gov/entrez/query.fcgi, 1966 to date. Limits imposed "English," "clinical trial," "meta analysis," "randomized controlled trial," "human," for specificity

- EMBASE, http://www.datastarweb.com, 1974 to date. Search term "(rituximab AND low grade lymphoma) AND (LG=EN) AND $((\mathrm{HUMAN}=\mathrm{YES}))$ "

- BIOSIS, http://www.datastarweb.com. Search term "(rituximab AND low grade lymphoma) AND (LG=EN) AND ((HUMANS\#))"

- Database of Abstracts of Reviews of Effects (DARE), National Health Service (NHS) Economic Evaluations Database (NHSEED), Health Technology Assessment (HTA), http://www.york.ac.uk/inst/crd/crddatabases.htm. All three databases searched together. All fields searched

- NHS HTA, http://www.ncchta.org

- National Guideline Clearinghouse, http://www.guideline.gov

- National Institute for Health and Clinical Excellence (NICE), http://www.nice.org.uk

- Cochrane Database of Systematic Reviews (CDSR), http://www.cochrane.org/index0.htm. Entire site searched

- Clinical Evidence (BMJ), http://www.clinicalevidence.com

- Clinical trials databases, http://www.clinicaltrials.gov, and http://www.clinicalstudyresults.org

Online abstracts from the American Society of Hematology 2005 and 2006 annual meetings were searched using the search terms "rituximab," "indolent NHL," or "cost effectiveness." Only articles directly related to rituximab maintenance were included. Following removal of out-of-scope articles, one paper was included.
Searching using the terms "follicular" or "indolent lymphoma" identified a further eight papers and four abstracts (Table 1).

\section{Table 1 | Evidence base included in the review}

\begin{tabular}{|lcc|}
\hline Category & \multicolumn{2}{c|}{ Number of records } \\
\cline { 2 - 3 } & Full papers & Abstracts \\
\hline Initial search & 94 & 81 \\
records excluded & 93 & 81 \\
records included & 1 & 0 \\
Additional studies identified & 8 & 4 \\
Total records included & 9 & 4 \\
Level 1 clinical evidence & 0 & 0 \\
(systematic review, meta analysis) & & 1 \\
Level 2 clinical evidence (RCT) & 4 & 1 \\
Level $\geq 3$ clinical evidence & 5 & 1 \\
trials other than RCT & 5 & 0 \\
$\quad$ case reports & 0 & 2 \\
Economic evidence & 0 & \\
\hline For definitions of levels of evidence, see Editorial Information on inside back cover. \\
RCT, randomized controlled trial.
\end{tabular}

\section{Disease overview}

Non-Hodgkin's lymphoma (NHL) is the sixth most common malignancy in men and women accounting for $4 \%$ and $3 \%$, respectively, of all new diagnoses of cancer in the UK (Cancer Research UK 2007). FL is the second most common form of NHL accounting for $22 \%$ of $\mathrm{NHL}$ in adults (Armitage \& Weisenburger 1998). Its incidence has been increasing over the past few decades by approximately $3 \%$ per year, but more recently the incidence has stabilized (Morton et al. 2006). FL is generally considered to be an incurable illness. It is characterized by periods of remission followed by episodes of relapse until, ultimately, the disease becomes resistant to therapy and death due to progressive disease ensues. The median survival of patients with advanced disease in the pre-rituximab era was 8-10 years. There is evidence that this has improved since the incorporation of rituximab into treatment regimens (Fisher et al. 2005; Swenson et al. 2005). The therapeutic strategy is to control the disease when necessary with the aim of maximizing the patients' quality of life as well possibly improving survival. 


\section{Current therapy options}

Asymptomatic patients with advanced disease who have reasonable bone marrow function can often be managed expectantly for a median of 2.5 years before systemic therapy is required. This "watch and wait" approach has been shown to be no worse than starting immediate systemic therapy with regards to survival, and is generally thought to result in a better quality of life by avoiding the side effects of chemotherapy (Ardeshna et al. 2003). When patients become symptomatic due to disease progression, systemic therapy is required. Prior to the introduction of rituximab, the choice of therapy included monotherapy with alkylating agents (e.g. chlorambucil or cyclophosphamide), multiagent chemotherapy with or without anthracyclines [e.g. CVP (cyclophosphamide, vincristine, prednisone), CHOP (cyclophosphamide, vincristine, prednisone, doxorubicin)], and purine analogs (e.g. fludarabine), either alone or in combination with alkylating agents \pm anthracycline. When patients relapse the same therapy is often used again if the duration of response is more than 2 years and there are no doselimiting considerations (e.g. maximum tolerated anthracycline dose), otherwise an alternative treatment is used. In some countries such as France, interferon alfa in induction or maintenance therapy is standard. Consolidation of second or subsequent remission with autologous stem cell transplantation (ASCT) has been shown to be superior to observation in terms of progression-free survival (PFS) and overall survival (OS) in a small, multicenter, randomized trial (Schouten et al. 2003) and is standard practice in some centers. Allogeneic stem cell transplantation appears to have curative potential but with significant risk limiting its use to selected younger patients (van Besien et al. 2003). Radiotherapy is very useful for the treatment of symptomatic nodes.

With the introduction of rituximab, therapeutic options have been considerably widened. Rituximab is a humanized, chimeric, monoclonal antibody directed against CD20. CD20 is a molecule expressed on normal and malignant B cells but not expressed on stem cells or plasma cells. CD20 is not internalized or shed and appears to be an ideal target for immunotherapy. Rituximab binding to CD20 results in cell death via several mechanisms, namely antibody-dependent cell-mediated cytotoxicity, complement-mediated cell lysis, and direct triggering of apoptosis by activation of intracellular signal transduction pathways. The specificity of rituximab accounts for its lack of toxicity.

In a pivotal trial, four doses of rituximab $375 \mathrm{mg} / \mathrm{m}^{2}$ per week were administered to 166 patients with relapsed or refractory indolent lymphoma, including 130 patients with follicular lymphoma (McLaughlin et al. 1998). The response rate was $48 \%$ (60\% for patients with $\mathrm{FL}$ ), and the median time to progression was 13 months. Toxicity was minimal and largely consisted of National Cancer Institute ( $\mathrm{NCl}$ ) grade 1 and 2 infusion-related side effects.

In another study, 49 patients with previously untreated, low tumor burden FL received this standard induction with rituximab (four weekly doses of rituximab $375 \mathrm{mg} / \mathrm{m}^{2}$ ) (McLaughlin et al. 1998). This trial has recently been updated after 7 years' followup (Colombat et al. 2006). The overall best response rate was 74\% [complete remission (CR) 50\%; partial remission (PR) 24\%]. The median PFS was 23.5 months with a median response duration of 28.6 months. A total of $24 \%$ of responders maintained their response at 5 years and $15 \%$ at 7 years without any further therapy.

Several phase III studies have shown that the addition of rituximab to induction chemotherapy (in relapsed and previously untreated patients) significantly improves PFS and in some cases an OS benefit has been demonstrated without a clinically significant increase in toxicity (Herold et al. 2004; Salles et al. 2004; Hiddemann et al. 2005; Marcus et al. 2005; Forstpointner et al. 2006; van Oers et al. 2006).

\section{Unmet needs}

Lymphoma cells that persist after induction therapy are responsible for subsequent relapse. Reduction in the numbers of these cells may result in prolongation of remissions. This reduction can be attempted by the administration of further consolidation therapy or by using a maintenance strategy. Consolidation therapy (e.g. high-dose therapy followed by ASCT) is aimed at eliminating as much residual disease as possible. Maintenance therapy on the other hand is administered in regular doses over a prolonged period of time to prevent the reestablishment of malignant cells and thereby prolong the time until relapse (Berinstein 2006). Maintenance therapy in $\mathrm{FL}$ is aimed at maximizing the patient's quality of life as well as improving survival. In order to achieve this, maintenance therapy should be convenient, nontoxic, and efficacious. As a maintenance therapy interferon alfa is inconvenient as it requires injections 3 times a week. It has significant side effects requiring temporary or permanent cessation of treatment, and in a meta analysis was found to be ineffective when administered as maintenance therapy (Rohatiner et al. 2005).

Rituximab, however, appears more suited as a maintenance therapy. It has minimal toxicity and requires infrequent dosing. In the pivotal study the peak plasma concentration of rituximab increased after each of the four infusions and the plasma clearance correspondingly decreased from $38.2 \mathrm{~mL} /$ hour after the first infusion to $9.2 \mathrm{~mL} /$ hour after the fourth infusion (McLaughlin et al. 1998). In $63 \%$ of patients rituximab was still detectable in the serum 3 months after the last infusion. Importantly, there was a significant positive correlation between median serum antibody levels and response (Berinstein et al. 1998). Responders had a median serum rituximab level of $25.4 \mathrm{mcg} / \mathrm{mL}$ compared with $5.9 \mathrm{mcg} / \mathrm{mL}$ in nonresponders. This finding and the observation that in some patients the duration of response to a second course of rituximab may be longer than the duration of response to the first course of rituximab suggested that a prolonged course of rituximab may improve response duration as well as improving remission rates (Davis et al. 2000). 
Table 2 | Results of randomized studies of rituximab maintenance in follicular lymphoma

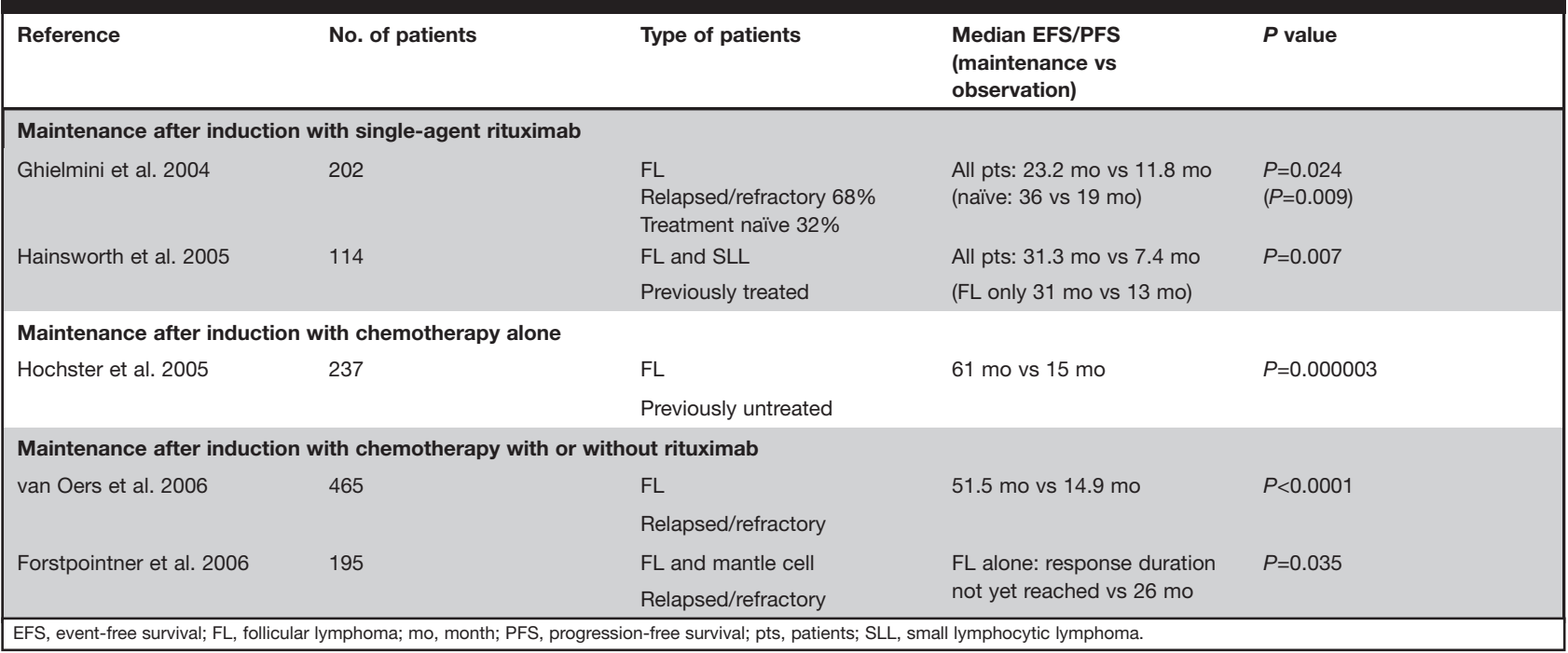

\section{Clinical evidence with rituximab maintenance}

The results of randomized studies of rituximab as maintenance therapy in follicular NHL are shown in Table 2.

\section{Rituximab maintenance after induction using single-agent rituximab}

In a phase II study, 62 patients with previously untreated indolent NHL [small lymphocytic lymphoma (SLL) 39\%, and FL 61\%] received rituximab $375 \mathrm{mg} / \mathrm{m}^{2}$ per week intravenously for 4 weeks (Hainsworth et al. 2002). A total of $77 \%$ were thought to require some therapy by the treating physician. Responders and those with stable disease (SD) at 6 weeks received repeated courses at 6-month intervals for a maximum of four courses or disease progression. At 6 weeks, responses were seen in $47 \%$ (CR 7\%, PR 40\%) and SD was observed in $45 \%$ of patients after induction. Fifty-eight percent went on to receive all four courses. Maintenance rituximab increased the overall response rate (ORR) to $73 \%$ with $37 \%$ achieving a CR. The median PFS was 34 months, compared with the 23.5 months achieved with rituximab induction alone in the study performed in asymptomatic patients with low tumor burden described above (Colombat et al. 2001).

Subsequently, two randomized studies of rituximab maintenance following standard rituximab induction have been reported showing a PFS/event-free survival (EFS) benefit in the maintenance arm. In the first study, performed by the Swiss Group for Clinical Cancer Research (SAKK), Ghielmini et al. (2004) treated 202 patients with chemotherapy-naïve (32\%) or relapsed/refractory (68\%) FL. None of the patients had received rituximab previously. In 185 evaluable patients the ORR at 12 weeks was $52 \%$ (67\% in chemotherapy-naïve and $46 \%$ in previously treated patients) with a CR rate of $8 \%$. One hundred and fifty-one patients with CR, PR, or SD at 12 weeks were randomized to no further treatment or prolonged treatment with rituximab with one further infusion given at month $3,5,7$, and 9 . There was a gradual decline in the ORR in the first group from $67 \%$ at 3 months to $44 \%$ at 12 months, and $28 \%$ at 24 months. In the group receiving rituximab maintenance the ORR remained stable initially (62\% at 3 months and $66 \%$ at 12 months) before declining at a slower rate to $45 \%$ at 24 months. The ORR was only significantly different between the two groups beyond 1 year $(P=0.046)$. The $\mathrm{CR}$ rate in the maintenance group increased from $12 \%$ to $29 \%$ in the maintenance arm but CR rates also rose in the control arm such that there was never a significant difference in $\mathrm{CR}$ rates between the two arms. With a median follow-up of 36 months the median EFS in the maintenance arm was almost double that in the control arm (23.2 vs 11.8 months, $P=0.024)$. This was more marked when responders to rituximab induction were considered separately ( 36 vs 16 months, $P=0.004$ ). Conversely there was no significant difference in EFS between the two arms when only those who achieved stable disease after rituximab induction were analyzed (11 vs 8 months, $P=0.35$ ). When only chemotherapy-naïve patients were considered, the EFS was 36 vs 19 months $(P=0.009)$. The median EFS of 3 years which was achieved with induction using single-agent rituximab followed by rituximab maintenance is comparable to the time to treatment failure (TTF) achieved with induction therapy with CHOP chemotherapy (30 months) (Hiddemann et al. 2005) or CVP plus rituximab (R-CVP) chemoimmunotherapy (27 months) (Marcus et al. 2005). Toxicity was acceptable with no increase in infections in the rituximab maintenance group despite a prolonged reduction in immunoglobulin $\mathrm{M}$ and $\mathrm{B}$ cell levels.

In the second study the primary endpoint was duration of rituximab benefit. One hundred and fourteen previously treated patients with FL and SLL received induction with four weekly doses of rituximab $375 \mathrm{mg} / \mathrm{m}^{2}$ (Hainsworth et al. 2005). None of the patients had received rituximab previously. Ninety patients 
(78\%) had an objective response (28\%) or SD (51\%) at 6 weeks and were randomized to the maintenance arm with four weekly doses of rituximab $375 \mathrm{mg} / \mathrm{m}^{2}$ every 6 months for a total of four treatments, or the re-treatment arm where further four weekly doses of rituximab were given only when disease progression was noted. In the re-treatment arm further reinduction with rituximab was allowed at subsequent episodes of disease progression provided the preceding duration of response was greater than 3 months.

When considering the patients with $\mathrm{FL}$ alone, the initial ORR was similar in each arm at $47 \%$ and $46 \%$. Unlike the SAKK study the best $\mathrm{CR}$ rate achieved was significantly better in the maintenance arm $(32 \%$ vs $7 \%)$. In common with the SAKK study, the median PFS was significantly better in the maintenance arm (31 vs 13 months). There was, however, no significant difference in duration of rituximab benefit between the maintenance arm and the re-treatment arm (31 vs 35 months). The re-treatment group used $29 \%$ less rituximab than the maintenance group to achieve this, which suggests an advantage for this group. However, at the time of reporting, significantly more patients in the re-treatment group remained on study (14 vs 4 ) and the disparity in rituximab usage is likely to decrease with further follow-up. In addition, the duration of rituximab benefit in the maintenance arm is likely to have been underestimated as the duration of maintenance therapy was limited in this arm but the number of re-treatment courses was not limited in the re-treatment arm. In fact, a followup study has shown that $33 \%$ of patients who progressed following 2 years of maintenance obtained a further response with single-agent rituximab while an additional $58 \%$ had stable disease (Hainsworth et al. 2006). Although questions remain about the validity of the primary endpoint, the benefit of rituximab maintenance in terms of improved PFS is not in doubt. Unfortunately, in common with the SAKK trial, there was no assessment of the patients' quality of life in this study. Hopefully the ongoing Eastern Cooperative Oncology Group (ECOG) 4402 RESORT trial (Rituximab Extended Schedule Or Retreatment Trial) will conclusively answer whether the re-treatment or maintenance results in a longer period of rituximab benefit. In this study previously untreated patients with low tumor burden, low-grade lymphomas will receive four doses of rituximab given at weekly intervals. Responders will be randomized to either receive re-treatment with further courses of rituximab (four doses given at weekly intervals) provided the time to progression is more than 6 months, or maintenance rituximab given as one infusion every 12 weeks until progression or unacceptable toxicity.

\section{Rituximab maintenance after induction using chemotherapy with or without rituximab}

Further improvement in PFS was anticipated if rituximab maintenance was administered after induction therapy using chemotherapy or chemotherapy combined with rituximab. Three randomized studies have now been published which confirm this hypothesis; two studies were performed in previously treated patients (Forstpointner et al. 2006; van Oers et al. 2006) and one study in previously untreated patients (Hochster et al. 2005).
In the European Organisation for Research and Treatment of Cancer (EORTC) 20981 Intergroup study, 465 patients with relapsed/refractory FL were reported (van Oers et al. 2006). Patients had received a maximum of two nonanthracyclinecontaining regimens prior to study entry $(80 \%$ had received one prior therapy), $16 \%$ had not responded to previous therapy, and prior exposure to rituximab was not allowed. Seventy percent of patients had an FL International Prognostic Index (FLIPI) score of two or more at diagnosis (Solal-Celigny et al. 2004). The study design involved two randomizations. Patients were initially randomized to receive $\mathrm{CHOP}$ or rituximab plus $\mathrm{CHOP}$ (R-CHOP) induction chemotherapy. Response was assessed after three courses, patients with PD or SD went off-study, and responders went on to receive a further three courses of induction therapy. A second randomization took place at completion of induction therapy between observation and maintenance rituximab (administered once every 3 months for a maximum of 2 years). The primary endpoint of the first randomization was response while that of the second randomization was PFS. The study was stopped early after a preplanned interim analysis showed that both primary endpoints had been achieved. Subgroup analysis, as requested by the independent data monitoring committee, demonstrated benefit of maintenance rituximab in the CHOP group but not in the R-CHOP group. It was suggested that the trial be amended with all patients receiving $\mathrm{R}-\mathrm{CHOP}$ induction followed by randomization to rituximab maintenance or not. Recruitment was therefore suspended pending a major protocol amendment. Meanwhile all data were collected and a final analysis performed. This process took 19 months by which time further follow-up was available for analysis. At this analysis it was found that the primary question of the amended protocol had already been answered and the trial was not reopened.

The ORR was significantly better in the group that received $\mathrm{R}-\mathrm{CHOP}$ induction ( $85.1 \%$ vs $72.3 \%, P<0.0001$ ), as was the CR rate $(29.5 \%$ vs $15.6 \%, P<0.0001)$; PR rates were similar $(55.6 \%$ vs $56.7 \%$ ). With a median follow-up of 39.4 months from first randomization there was a significant increase in median PFS in the R-CHOP group (33.1 vs 20.2 months, $P=0.003$ ) without a significant increase in OS at 3 years $(82.5 \%$ vs $71.9 \%, P=0.096)$. A total of 334 patients were randomized to receive maintenance rituximab or not. With a median follow-up from second randomization of 33.3 months, the median PFS was significantly greater in the rituximab maintenance group (51.5 vs 14.9 months, $P<0.0001$ ), equating to a gain of 3 years in PFS. A gain in PFS of approximately 2.5 years was present whether patients received CHOP induction (42.2 vs 11.6 months, $P<0.0001$ ) or $\mathrm{R}-\mathrm{CHOP}$ induction ( 51.8 vs 23 months $P=0.0043$ ), indicating that the antilymphoma effect of rituximab is not exhausted during induction. Importantly, rituximab maintenance was also found to confer a significant OS benefit at 3 years when compared with observation, with the risk of dying being reduced significantly (85.1\% vs $77.1 \%, P=0.0111$ ). No breakdown of OS benefit conferred by rituximab maintenance according to induction regimen was given and therefore there is no evidence that rituximab maintenance conferred a significant OS benefit compared with observation if patients had received $\mathrm{R}-\mathrm{CHOP}$ as 
induction. The authors produced some data which indicate that only a minority $(41 \%)$ of patients in the observation arm received rituximab as part of their first postprotocol treatment. Even though this figure is higher than the $16 \%$ of patients in the maintenance arm who received rituximab as part of their first postprotocol treatment, the fact that many patients in the observation arm did not receive rituximab with their next treatment may account for some of the observed OS benefit for patients in the maintenance arm.

Maintenance rituximab resulted in a nonsignificant increase in grade 3 and 4 neutropenia (10.8\% vs $5.4 \%$ ), which probably accounted for the significant increase in grade 3 and 4 infections (mainly ear, nose, and throat infections) in this group (9\% vs $2.4 \%$, $P=0.009$ ). Six of 167 patients withdrew from the maintenance arm because of toxicity; four due to infection.

It therefore appears that in patients with relapsed and refractory FL, maintenance rituximab improves median PFS more than three-fold when compared with observation. This is achieved with minimal increase in toxicity. Even when rituximab maintenance follows R-CHOP induction the median PFS is more than doubled. The study also demonstrates an OS benefit at 3 years with rituximab maintenance when patients who received $\mathrm{R}-\mathrm{CHOP}$ and CHOP induction are analyzed together. However this study provides no evidence of a significant OS benefit attributable to rituximab maintenance in the group who received $\mathrm{R}-\mathrm{CHOP}$ induction although the study was not designed to detect such a difference.

The German Lymphoma Study Group (GLSG) randomized patients with relapsed/refractory $\mathrm{FL}$ and mantle cell lymphoma to receive either FCM (fludarabine, cyclophosphamide, mitoxantrone) or rituximab plus FCM (R-FCM) as induction therapy (Forstpointner et al. 2006). Responders underwent a second randomization to observation or to receive a course of rituximab maintenance at 3 and 9 months. Each maintenance course consisted of four weekly doses of rituximab. Patients who had received prior rituximab were eligible but no information was given regarding the number of patients who had received rituximab prior to study entry. The first randomization was stopped after 147 patients were recruited as R-FCM was found to be superior in terms of response, response duration, and survival. Subsequent patients all received R-FCM induction. Results of the second randomization in the 81 patients with $\mathrm{FL}$ who received R-FCM induction demonstrated the benefit of rituximab maintenance compared with observation. After a median followup of 26 months the median response duration was 17 months in the observation arm but had not been reached in the maintenance arm $(P=0.035)$. No survival difference has yet been observed (3-year OS $57 \%$ vs $77 \%, P=0.1$ ). Infusion-related side effects were noticed in $8 \%$ of maintenance cycles, generally mild or moderate with only one patient requiring therapy to be discontinued due to a severe allergic reaction.

Both the EORTC and GLSG studies recruited patients with relapsed/refractory disease. In an ECOG study, 401 previously untreated patients with FL or SLL were recruited and received induction with six to eight cycles of CVP chemotherapy (Hochster et al. 2005). Responders and patients with SD were randomized to receive maintenance with four weekly doses of rituximab administered every 6 months for a maximum of four courses (i.e. 2 years). Two hundred and thirty-seven patients with FL were recruited, $64 \%$ of whom had high tumor burden and $37 \%$ had high-risk disease according to the FLIPI. There was a highly significant difference in 4-year PFS between the maintenance and observation group (56\% vs $33 \%, P=0.0000003)$, and the median PFS was increased by almost 4 years in the maintenance group (61 vs 15 months). This study also revealed an OS benefit with rituximab maintenance at 4 years compared with observation (88\% vs $72 \%, P=0.03$ ).

Unfortunately none of the randomized studies of maintenance rituximab have measured patient-related quality of life. It is generally assumed that a patient has a better quality of life if periods of remission are prolonged; however, for some patients repeated visits to receive therapy when they are otherwise well can be a constant reminder of their disease and actually have a significant negative impact on their quality of life. If rituximab is given every 2 or 3 months the infusion can be given on the same day as a scheduled follow-up visit thus minimizing the disruption to the patient's life.

Most patients now receive rituximab combined with chemotherapy as first-line therapy and the value of maintenance in this setting has not been demonstrated as yet. It is noteworthy that CVP followed by rituximab maintenance in the ECOG study yields a PFS almost twice the 33 months achieved by front-line R-CVP induction chemotherapy alone in the study by Marcus et al. (2005). This suggests rituximab maintenance following R-CVP in previously untreated patients with FL is likely to be of benefit, however it must be noted that the proportion of patients with high-risk FLIPI scores were different in the two studies $37 \%$ of patients were high risk in the ECOG study compared with $45 \%$ in the Marcus et al. study).

The value of rituximab maintenance following first-line therapy with a rituximab chemotherapy combination will be answered when the results of the PRIMA study are available. In this Groupe d'Etude des Lymphomes de l'Adulte (GELA)-sponsored study, which closed to recruitment in spring 2007, patients received a choice of induction chemotherapy combined with rituximab, and responders were then randomized to observation or maintenance rituximab $375 \mathrm{mg} / \mathrm{m}^{2}$ given once every 2 months for a maximum of 2 years. The primary endpoint is PFS. The single-arm MAXIMA study is also currently recruiting patients who have had a response to rituximab-containing induction therapy. These patients, who may have had previous therapy, are allocated rituximab $375 \mathrm{mg} / \mathrm{m}^{2}$ every 2 months for 2 years. The main aim is to assess safety in a wider patient population.

\section{Economic evidence and resource utilization}

The standard therapeutic approach following induction of a remission in patients with relapsed or refractory $\mathrm{FL}$ is either to consolidate with high-dose therapy followed by ASCT or to keep 
the patient under observation and to re-treat when relapse of disease progression occurs. Evidence for economic benefit, or otherwise, of rituximab maintenance compared with either ASCT or observation followed by re-treatment is very limited.

A Finnish study reported only in abstract form compared the direct medical costs of ASCT to that of maintenance rituximab (eight doses over 2 years) (Leppä et al. 2006). The cost of the transplant was calculated to be $€ 38600$ while the cost of maintenance rituximab was $€ 19700$. ASCT resulted in a median PFS of 34.1 months $(n=12)$. When this was compared to the 51.9 months achieved in the EORTC 20981 study, rituximab maintenance was shown to improve PFS by 17.8 months. On the basis of this limited data the authors concluded that improved PFS with cost savings may be achieved with the use of rituximab maintenance rather than ASCT. Clearly the characteristics of the patients who underwent ASCT need to be comparable to those recruited into the EORTC study for this comparison to be valid and it is not clear that this was the case. In the CUP study (Schouten et al. 2003), which demonstrated the benefit of ASCT compared with observation in patients with relapsed FL (not refractory patients), the median PFS had not been reached with a follow-up of 69 months. Clearly, if the PFS for ASCT had been based on this study the outcome may have been different.

Maturi et al. (2006) developed a pharmacoeconomic model based on the EORTC data and quality-of-life data obtained by questionnaire from 165 patients with $\mathrm{FL}$ not included in the EORTC study. According to this analysis the incremental costeffectiveness ratio of rituximab maintenance versus observation alone was estimated at \$Can20428 per quality-adjusted life-year gained.

In the study by Hainsworth et al. (2005) no significant difference in the duration of the period of rituximab benefit was noted when maintenance was compared with re-treatment. Re-treatment used $29 \%$ less rituximab compared with maintenance. This study did have some shortcomings as mentioned earlier and no consideration was given to the patients' quality of life.

\section{Patient group/population}

Rituximab maintenance in patients with relapsed and refractory FL only appears to benefit patients who respond to induction therapy. In the SAKK study, only responders to single-agent rituximab at 12 weeks benefited; those with SD following induction did not. In the study by Hainsworth et al. (2005) there was no analysis of the benefit of rituximab maintenance according to the response (CR/PR vs SD) to rituximab induction. In the EORTC and GLSG studies only responders to induction therapy were eligible for randomization to maintenance; in the EORTC study both patients who achieved a CR and those who achieved a PR to induction therapy had a significantly improved PFS with maintenance therapy.

Rituximab maintenance following first-line chemotherapy combined with rituximab is widely expected to result in a significant benefit for patients in terms of PFS but, as yet, this remains unproven.

\section{Dosage, administration, and formulation}

Rituximab is available in $100 \mathrm{mg}$ concentrate in $10 \mathrm{~mL}$ for intravenous infusion. The recommended dose of rituximab used as a single agent for adult patients with $\mathrm{FL}$ is $375 \mathrm{mg} / \mathrm{m}^{2}$ body surface area, administered as an intravenous infusion once weekly for 4 weeks. The recommended dosage of rituximab in combination with CVP chemotherapy for adults with $\mathrm{FL}$ is $375 \mathrm{mg} / \mathrm{m}^{2}$ for eight cycles (21 days/cycle), administered on day 1 of each chemotherapy cycle. Premedication consisting of an antipyretic and an antihistamine [e.g. acetaminophen (paracetamol) and diphenhydramine] should always be administered before each infusion of rituximab, and premedication with glucocorticoids should also be considered. Patients should be closely monitored.

\section{Place in therapy}

There is clear evidence from randomized studies that rituximab maintenance prolongs PFS substantially in patients with relapsed/refractory $\mathrm{FL}$ who are rituximab naïve and respond to reinduction therapy with either single-agent rituximab, CHOP chemotherapy with or without rituximab, or FCM with rituximab. There is also evidence from one randomized study that rituximab maintenance prolongs PFS and OS in previously untreated patients who do not progress following induction with CVP chemotherapy (without rituximab). It is not clear whether patients with SD after CVP have a significant improvement in PFS and OS. There is substantial evidence from one randomized study indicating that rituximab maintenance significantly improves EFS in previously untreated patients who receive single-agent rituximab as induction therapy. There is moderate evidence that rituximab maintenance improved OS in a group of patients with relapsed/refractory $\mathrm{FL}$ who were rituximab naïve and who responded to reinduction with a combination of either $\mathrm{CHOP}$ chemotherapy with rituximab or CHOP chemotherapy alone. There is however currently no evidence from randomized studies that unequivocally demonstrates an OS benefit from rituximab maintenance in patients with relapsed/refractory FL who receive only rituximab-containing chemotherapy at reinduction.

There is moderate evidence to suggest that in patients with relapsed/refractory $\mathrm{FL}$, re-treatment with rituximab at disease progression may confer a similar period of rituximab benefit as rituximab maintenance.

There is currently no evidence from randomized studies that clearly demonstrates the benefit of rituximab maintenance in patients with relapsed/refractory FL who have been exposed to rituximab prior to relapse, in previously untreated patients with FL who receive chemotherapy and rituximab as induction therapy, or for an improvement in patients' quality of life.

Taken together, currently the place of rituximab maintenance in the therapy of FL appears to be in two main groups of patients. 
Firstly, a dwindling group of patients with relapsed/refractory disease who have not received rituximab previously and who are not suitable for or who do not wish to undergo ASCT. This group will get smaller and smaller as more patients receive rituximab chemotherapy combinations as first-line therapy. The large improvement in PFS seen with rituximab maintenance may be comparable to that achieved in the same patient population following consolidation therapy with ASCT. Whether one treatment is superior can only really be determined by further randomized studies. The results of such studies, if they take place, may soon become obsolete if the place of rituximab maintenance is found to be in a first remission following first-line therapy with rituximab chemotherapy combinations.

The second group includes patients who receive first-line therapy using chemotherapy without rituximab. Now that rituximab is widely available and rituximab chemotherapy combinations have been shown to be superior to chemotherapy alone as induction therapy, patients are unlikely to be treated in this way.

Many different schedules of rituximab maintenance have been used. It is not clear which schedule is optimal. Gordan et al. (2005) treated patients with rituximab $375 \mathrm{mg} / \mathrm{m}^{2}$ weekly for 4 weeks. Further rituximab was administered only when the plasma concentration fell below $25 \mathrm{mcg} / \mathrm{mL}$ (a level which correlated with response in a previous study). The median time to the next infusion to achieve this was 2-5 months, with $50 \%$ of patients achieving this level with a dosing interval of 3 months and almost all with a dosing interval of 2 months. This suggests that $2-3$ monthly dosing is probably optimal.

The duration of maintenance is also uncertain. Further rituximab benefit has been noticed even after 2 years. However it is worth bearing in mind that in patients with low tumor burden who received rituximab $375 \mathrm{mg} / \mathrm{m}^{2}$ weekly for 4 weeks, $24 \%$ of responders maintained their response at 5 years and $15 \%$ at 7 years without any further therapy (Colombat et al. 2006). Of course, the longer the maintenance period the greater the financial cost as well as the possibility that continued $B$-cell depletion will result in significant adverse effects. The SAKK is currently running a study comparing two different schedules of rituximab maintenance in patients with untreated, relapsed, or refractory $\mathrm{FL}$. After standard single-agent rituximab $375 \mathrm{mg} / \mathrm{m}^{2}$ induction patients will be randomized to receive either one dose of rituximab $375 \mathrm{mg} / \mathrm{m}^{2}$ every 2 months on four occasions, or every 2 months for 5 years or until relapse, progression, or unacceptable toxicity. This study should give a greater insight into the adverse effects of rituximab maintenance.

\section{Acknowledgments}

Dr Ardeshna has participated in two advisory boards, and received honoraria and travel expenses from F. Hoffmann-La Roche. Dr Ardeshna is the chief investigator of the "watch and wait" study, which is funded by a grant from F. Hoffmann-La Roche.

\section{References}

Ardeshna KM, Smith P, Norton A, et al; British National Lymphoma Investigation. Long-term effect of a watch and wait policy versus immediate systemic treatment for asymptomatic advanced-stage non-Hodgkin lymphoma: a randomised controlled trial. Lancet. 2003;362:516-522.

Armitage JO, Weisenburger DD. New approach to classifying non-Hodgkin's lymphomas: clinical features of the major histologic subtypes. Non-Hodgkin's Lymphoma Classification Project. J Clin Oncol. 1998;16:2780-2795.

Berinstein NL, Grillo-López AJ, White CA, et al. Association of serum rituximab (IDEC-C2B8) concentration and anti-tumor response in the treatment of recurrent low-grade or follicular non-Hodgkin's lymphoma. Ann Oncol. 1998;9:995-1001.

Berinstein NL. Principles of maintenance therapy. Leuk Res. 2006;30 (Suppl. 1):S3-S10.

Cancer Research UK. UK cancer incidence statistics. Available at: http://info.cancerresearchuk.org/cancerstats/incidence/?a=5441 (accessed June 2007)

Colombat P, Salles G, Brousse N, et al. Rituximab (anti-CD20 monoclonal antibody) as single first-line therapy for patients with follicular lymphoma with a low tumor burden: clinical and molecular evaluation. Blood. 2001;97:101-106.

Colombat P, Brousse N, Morschhauser, et al. Single treatment with rituximab monotherapy for low-tumor burden follicular lymphoma: survival analyses with extended follow-up of 7 years. Blood. 2006;108. Abstract 486.

Davis TA, Grillo-López AJ, White CA, et al. Rituximab anti-CD20 monoclonal antibody therapy in non-Hodgkin's lymphoma: safety and efficacy of retreatment. J Clin Oncol. 2000;18:3135-3143.

Fisher RI, LeBlanc M, Press OW, Maloney DG, Unger JM, Miller TP. New treatment options have changed the survival of patients with follicular lymphoma. J Clin Oncol. 2005;23:8447-8452.

Forstpointner R, Unterhalt M, Dreyling M, et al; German Low Grade Lymphoma Study Group (GLSG). Maintenance therapy with rituximab leads to a significant prolongation of response duration after salvage therapy with a combination of rituximab, fludarabine, cyclophosphamide, and mitoxantrone (R-FCM) in patients with recurring and refractory follicular and mantle cell lymphomas: results of a prospective randomized study of the German Low Grade Lymphoma Study Group (GLSG). Blood. 2006;108:4003-4008.

Ghielmini M, Schmitz SF, Cogliatti SB, et al. Prolonged treatment with rituximab in patients with follicular lymphoma significantly increases event-free survival and response duration compared with the standard weekly $\times 4$ schedule. Blood. 2004;103:4416-4423.

Gordan LN, Grow WB, Pusateri A, Douglas V, Mendenhall NP, Lynch JW. Phase II trial of individualized rituximab dosing for patients with CD20-positive lymphoproliferative disorders. J Clin Oncol. 2005;23:1096-1102.

Hainsworth JD, Litchy S, Burris HA, et al. Rituximab as first-line and maintenance therapy for patients with indolent non-Hodgkin's lymphoma. J Clin Oncol. 2002;20:4261-4267.

Hainsworth JD, Litchy S, Shaffer DW, Lackey VL, Grimaldi M, Greco FA. Maximizing therapeutic benefit of rituximab: maintenance therapy versus retreatment at progression in patients with indolent non-Hodgkin's lymphoma - a randomized phase II trial of the Minnie Pearl Cancer Research Network. J Clin Oncol. 2005;23:1088-1095.

Hainsworth JD, Meng C, Spigel DR, Raefsky EL, Barton JH, Greco FA. Long-term followup of patients with follicular lymphoma (FL) treated with two years of maintenance rituximab: response to rituximab retreatment at progression. Blood. 2006;108. Abstract 4723.

Herold M, Pasold R, Srock S, et al. Results of a prospective randomised open label phase III study comparing rituximab plus mitoxantrone, chlorambucile, prednisolone chemotherapy (R-MCP) versus $\mathrm{MCP}$ alone in untreated advanced indolent non-Hodgkin's lymphoma and mantle-cell-lymphoma. Blood. 2004;104. Abstract 584.

Hiddemann W, Kneba M, Dreyling M, et al. Frontline therapy with rituximab added to the combination of cyclophosphamide, doxorubicin, vincristine, and 
prednisone (CHOP) significantly improves the outcome for patients with advanced-stage follicular lymphoma compared with therapy with CHOP alone: results of a prospective randomized study of the German Low-Grade Lymphoma Study Group. Blood. 2005;106:3725-3732.

Hochster H, Weller E, Gascoyne RD, et al. Maintenance rituximab after CVP results in superior clinical outcome in advanced follicular lymphoma $(\mathrm{FL})$ : results of the E1496 phase III trial from the Eastern Cooperative Oncology Group and the Cancer and Leukemia Group B. Blood. 2005;106. Abstract 349.

Leppä S, Linna M, Nyman H, Taimela E. Cost-effectiveness of rituximab maintenance treatment versus autologous stem cell transplantation (ASCT) in patients with relapsed follicular lymphoma (FL). Blood. 2006;108. Abstract 3337.

Marcus R, Imrie K, Belch A, et al. CVP chemotherapy plus rituximab compared with CVP as first-line treatment for advanced follicular lymphoma. Blood. 2005;105:1417-1423.

Maturi B, Mikhael JR, Dunlop WCN, Tilden DT, Wong L. Maintenance therapy with rituximab for follicular lymphoma is cost-effective - a Canadian perspective. Blood. 2006;108. Abstract 343.

McLaughlin P, Grillo-López AJ, Link BK, et al. Rituximab chimeric anti-CD20 monoclonal antibody therapy for relapsed indolent lymphoma: half of patients respond to a four-dose treatment program. J Clin Oncol. 1998;16:2825-2833.

Morton LM, Wang SS, Devesa SS, Hartge P, Weisenburger DD, Linet MS. Lymphoma incidence patterns by WHO subtype in the United States, 1992-2001. Blood. 2006;107:265-276.

Rohatiner AZ, Gregory WM, Peterson B, et al. Meta-analysis to evaluate the role of interferon in follicular lymphoma. J Clin Oncol. 2005;23:2215-2223.

Salles GA, Foussard C, Nicolas M, et al. Rituximab added to alfalFN+CHVP improves the outcome of follicular lymphoma patients with a high tumor burden: to first analysis of the GELA-GOELAMS FL-2000 randomized trial in 359 patients. Blood. 2004;104. Abstract 160.

Schouten HC, Qian W, Kvaloy S, et al. High-dose therapy improves progression-free survival and survival in relapsed follicular non-Hodgkin's lymphoma: results from the randomized European CUP trial. $J$ Clin Oncol. 2003;21:3918-3927.

Solal-Céligny $\mathrm{P}$, Roy $\mathrm{P}$, Colombat $\mathrm{P}$, et al. Follicular lymphoma international prognostic index. Blood. 2004;104:1258-1265.

Swenson WT, Wooldridge JE, Lynch CF, Forman-Hoffman VL, Chrischilles E, Link BK. Improved survival of follicular lymphoma patients in the United States. J Clin Oncol. 2005;23:5019-5026.

van Besien K, Loberiza FR, Bajorunaite R, et al. Comparison of autologous and allogeneic hematopoietic stem cell transplantation for follicular lymphoma. Blood. 2003;102:3521-3529.

van Oers MH, Klasa R, Marcus RE, et al. Rituximab maintenance improves clinical outcome of relapsed/resistant follicular non-Hodgkin lymphoma in patients both with and without rituximab during induction: results of a prospective randomized phase 3 intergroup trial. Blood. 2006;108:3295-3301.

Correspondence: Dr Kirit M.Ardeshna, University College Hospital, London \& Mount Vernon Cancer Centre, Northwood, UK, or at kirit.ardeshna@uclh.nhs.uk 